\title{
Hans Selye y la endocrinología social
}

\author{
Alfredo Jácome Roca
}

Internista-Endocrinólogo. Miembro Honorario, Asociación Colombiana de Endocrinología, Diabetes y Metabolismo. Miembro de Número, Academia Nacional de Medicina, Bogotá.

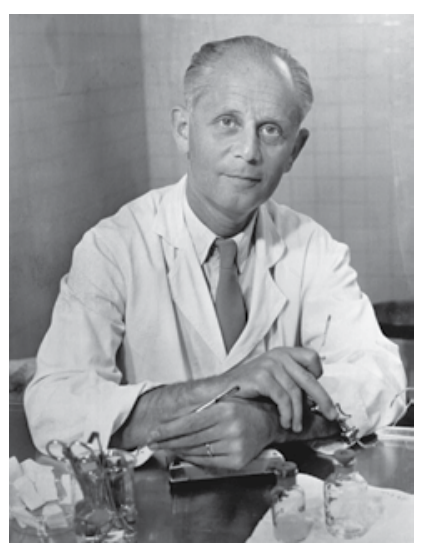

$\mathrm{L}$ a endocrinología se enriqueció enormemente por la descripción del síndrome general de adaptación, seguido de aquella teoría del estrés del húngaro Hans Selye, quien finalizaría sus días como canadiense por adopción.

Para su época de estudiante de medicina en la Universidad Alemana de Praga, este hijo de un cirujano del Imperio Austro-Húngaro empezó a observar que sus pacientes —además de los síntomas de la enfermedad por la cual consultaban- presentaban cansancio, pérdida del apetito y de peso, astenia y otras manifestaciones que hoy tal vez incluiríamos en el síndrome de fatiga crónica, algo que él llamaría "sentirse enfermo". Una vez graduado de médico, estudió en su universidad química orgánica, obteniendo un doctorado. Después fue becario de la Rockefeller en Johns Hopkins para estudios posdoctorales y luego pasó a la Universidad de McGill, donde se desempeñó como profesor de bioquímica.

Mientras trabajaba allí, uno de los proyectos del departamento consistía en administrar a ratones un macerado de ovario o de placenta, en la búsqueda de alguna nueva hormona. Los animales de experimentación respondían con cambios fisiopatológicos consistentes en hiperplasia de las glándulas suprarrenales, atrofia del timo y de órganos linfáticos y hemorragias, especialmente del tracto digestivo (por úlceras gástricas superficiales). Como sus intentos por purificar alguna sustancia hormonal causante de los cambios fallaron en su totalidad, no podía comprender en realidad qué estaba produciendo esta patología. Selye tomó entonces la decisión de ensayar otro tipo de productos o de sobrecargas al organismo, para ver su efecto agudo en el ratón. Lo observado ocupó la primera parte de una carta que envió al editor de la revista $\mathrm{Na}$ ture, publicada en mayo 18 de 1936, en una sola página, poco más de 500 palabras, a renglón seguido de cartas de otros au- tores y sin incluir una sola referencia ${ }^{(1)}$. Comenzaba así: Experimentos en ratas muestran que si el organismo es lesionado por agentes nocivos agudos no específicos tales como la exposición al frío, cirugía, producción de shock medular (sección medular), ejercicio muscular excesivo, o intoxicaciones con dosis subletales de diversos fármacos (adrenalina, atropina, morfina, formaldehído, etc.), aparece un síndrome típico, con síntomas independientes de la naturaleza del agente dañino o del tipo de fármaco empleado, que representan más bien una respuesta genérica al daño como tal.

Había entonces un común denominador en lo que se llama "estar enfermo". Acto seguido describió las etapas que consecutivamente se observaban en el ratón durante el experimento: una reacción inicial de alarma (que ahora sabemos que se asocia con la liberación de hormonas del estrés, al activarse el eje CRH-ACTH-Cortisol, contrarreguladoras de la insulina), un estado de resistencia y finalmente una fase de agotamiento ${ }^{(2)}$.

El investigador continúa con su resumida exposición, elucubrando sobre los fenómenos observados: Consideramos que la primera etapa es una expresión general de alarma del organismo cuando se enfrenta a una súbita situación crítica, y por tanto que lo denominamos "reacción general de alarma. Dado que el síndrome, como un todo, parece representar un esfuerzo generalizado del organismo para adaptarse a las nuevas situaciones, se le podría denominar "síndrome general de adaptación". Podría ser comparado con otras reacciones de defensa general tales como la inflamación o la formación de anticuerpos inmunológicos... nos parece que las formas pronunciadas de esta reacción de tres etapas representan la respuesta habitual del organismo a estímulos tales como cambios en la temperatura, efectos de las drogas, ejercicio muscular, etc., pudiendo luego ocurrir una habituación a la situación.

Esta respuesta fisiológica a estímulos externos la denominó luego "Respuesta al Stress" y finalmente "stress" (estrés), aunque de manera transitoria también recibió la denominación de "Síndrome de Selye". Más tarde, en el mismo año, publicó un artículo más largo en el British Journal of Experimental Pathology(2), en el que demostró que la involución del timo estaba mediada por las suprarrenales, ya que los animales adrenalectomizados no presentaban el fenómeno.

Selye no estuvo seguro durante años de si la palabra estrés era la correcta para describir sus hallazgos. El término puede usarse como sustantivo o verbo y tiene sus orígenes en el latín strictus (apretado, comprimido), en el inglés distress y en el francés estrece con significados similares. Por supuesto, la palabra estrés (de materiales) era usada desde siglos antes 
por ingenieros. Hemos oído por ejemplo el término "fatiga del metal", como explicación de algunos desastres aeronáuticos; esto no es otra cosa que por causa de la presión a que se somete el metal de los aviones, puede terminar cediendo después de varias décadas, causando hendiduras, desprendimientos y accidentes catastróficos. Cannon, su profesor, había usado la palabra en sus estudios sobre la homeostasis ${ }^{(3,4)}$. Cannon era un fisiólogo, interesado en la fisiología del sistema nervioso autónomo y en sus efectos sobre las vísceras, y en particular del aparato digestivo. Este sistema gastrointestinal le fue particularmente útil a Pavlov para demostrar su reflejo condicionado (soporte de una teoría neural sobre el control orgánico), pero también a Bayliss y a Starling con su teoría hormonal a partir del descubrimiento de la secretina. Los estudios con la epinefrina y el sistema nervioso autónomo habían progresado, de tal manera que se llegó a saber que las emociones estimulaban el sistema simpático, del que hacía parte la médula suprarrenal que liberaba epinefrina, ayudando en la respuesta de emergencia de la lucha o huída. Cannon había observado en sus experimentos con gatos que un susto o el miedo paralizaban el aparato digestivo por medio de la epinefrina (después se sabría que la acción beta de la epinefrina era inhibidora, mientras que la alfa de la norepinefrina era estimuladora). Es decir, ya estaba al tanto de lo que en la época se sabía de la epinefrina (que los laboratorios Parke-Davis popularizaron con su marca "Adrenalina") y había continuado con el estudio de los efectos de algunos de estos neurotransmisores, que dieron lugar entre otras cosas a la nueva ciencia de la farmacología ${ }^{(5)}$.

Otro fisiólogo que influyó en sus ideas investigativas fue Claude Bernard, quien desarrolló en el siglo XIX el concepto del "medio interno" — de donde saldría el término "glándulas de secreción interna" - por lo que muchos le consideran el padre de la endocrinología; esto a pesar del error cometido al considerar al hígado una de estas glándulas, y a la producción hepática de glucosa una secreción endocrina (la bilis era la secreción exocrina).

Otra anécdota — que tomé de la popular Wikipedia( ${ }^{(6)}$ habla de que "Selye vivía en una pensión con estudiantes de doctorados de ingeniería que solían mencionar en los descansos sus trabajos acerca del "strain", proceso de fatiga que sufren los materiales con el tiempo; Selye les dijo que algo parecido le ocurría con los ratones y que eso también sucedía con el resto de los seres vivos. Cuando publicó en "Science" un resumen de su trabajo con la palabra "stress", éstos lo felicitaron, tanto por publicar en esa importante revista, como por haber inventado aquella maravillosa palabra. Él sorprendido, dijo que no la había inventado, que era la palabra que ellos como ingenieros usaban para describir la fatiga que sucede en los materiales. Lo que había sucedido era que como su inglés aún no era perfecto se había confundido pensando que se decía "stress". Por su origen europeo, Selye sin embargo llegó a dominar 7 u 8 idiomas, y podía desempeñarse sin problemas en un país bilingüe como Canadá. En años posteriores se refirió a que también generan estrés las demandas de carácter social y las amenazas del en- torno del individuo que requieren de capacidad de adaptación. Es decir, que en estas personas también existe una "respuesta no específica del organismo a cualquier demanda exterior".

Varias enfermedades se relacionan con el estrés agudo, como úlceras gástricas, estados de shock, estrés postraumático y depresión obstétrica, estados posquirúrgicos, quemaduras e infecciones severas, mientras que otras podrían relacionarse con estrés crónico: trastornos disfuncionales del aparato digestivo que llevan a síntomas dispépticos o a intestino irritable, ansiedad generalizada, insomnio, cefalea tensional, disfunción eréctil, enfermedades cardiovasculares y tendencia a las adicciones; aunque su participación es menos clara en esos casos, el uso del término es bastante común ${ }^{(7-10)}$. En 1945 Selye dio un nuevo pero trascendente paso al dejar McGill para fundar el Instituto de Medicina y Cirugía Experimentales de la Universidad de Montreal. Dejó también una importante biblioteca donde coleccionó la explosión de trabajos investigativos que con el correr de los años - y aún en el presente y en el futuro- se hacen sobre las múltiples implicaciones del tema en todas las especialidades médicas, salud pública, filosofía, sociología y política.

Durante años, la teoría fue considerada atractiva, pero mirada con escepticismo. Aún en épocas de Selye, se le dio mucho manejo mediático, lo que hace que en la actualidad cualquier persona esté familiarizada con el término.

A pesar de su importante hallazgo, Selye no recibió el premio Nobel (tampoco su mentor J.B. Collip, quien ayudó al desarrollo de la insulina) aunque sí lo recibió uno de sus alumnos en Montreal, Roger Guillemin, por sus trabajos con péptidos liberadores hipotalámicos. Sin quedarse anclado en su investigación primigenia, Selye hizo extensos estudios de la estructura y actividad de los glucocorticoides en los años treinta y en los cuarenta ${ }^{(11,12)}$ y de allí salió una primera clasificación racional de los esteroides, que se dividieron en corticoides (provenientes de la corteza suprarrenal), testoides o andrógenos y foliculoides o estrógenos ${ }^{(13)}$. Reconoció las acciones anti y proinflamatorias de los gluco y mineralocorticoides en modelos animales, con los que siempre trabajó; inició la investigación en neuroesteroides con el hallazgo de que algunos de ellos eran potentes agentes anestésicos ${ }^{(14)}$. Cuando se otorgó el Nobel de Medicina en 1950 a los químicos Edward Kendall y Tadeus Reichstein y al clínico Philip Hench por el aislamiento y primeras aplicaciones terapéuticas de la cortisona ${ }^{(15)}$, Hans Selye era ya reconocido como autoridad mundial en campos como endocrinología, química de esteroides, cirugía experimental y patología. En su vida, publicó más de 1.500 artículos de investigación y revisión, fue autor único de 32 libros y entrenó a 40 doctores en ciencias biológicas, incluyendo al Nobel Guillemin. No es raro que la academia sueca haya premiado a científicos que investigan temas que producen soluciones prácticas o tratamientos concretos. En nuestro medio tuvo entrenamiento con Selye, Hernán Mendoza Hoyos, un clínico procedente del Hospital San Juan de Dios que también fue uno de los fundadores de la Asociación Colombiana de Endocrinología, Diabetes y Metabolismo (figura 1). 
Considerando que el término estrés es demasiado popular, y que la homeostasis debe dejarse para los parámetros bioquímicos cuya alteración puede rápidamente terminar con la vida $\left(\mathrm{pH}, \mathrm{pO}_{2}\right.$, glicemia, etc.) años más tarde McEwen postuló el nuevo término de "alostasis", definido como "la manutención de la estabilidad gracias a la adaptación a cambios esperados o inesperados"(16). Los organismos vivos tienen rutinas para conseguir y trasportar alimentos, aparearse, migrar, cambiar plumas, hibernar. La organización social trae ventajas para el suministro de servicios básicos y solidarios, pero trae desventajas como la jerarquización, competencia y subordinación, situaciones en las que sobrevive el más fuerte. Cuando la demanda estresante, externa o interna es superior a la capacidad de respuesta, se presenta la sobrecarga alostática, que se asocia con fenómenos y enfermedades como la depresión y otras manifestaciones psiquiátricas, la deposición abdominal de grasa (favorecida por hipercortisolismo); esto lleva a hiperinsulinismo, síndrome de ovarios poliquísticos, enfermedad cardiovascular, diabetes, hipertensión, exacerbación de asma bronquial, uso de sustancias adictivas (cigarrillo, alcohol, narcóticos), úlcera péptica, enfermedades autoinmunes, amenorrea hipotalámica, etc. ${ }^{(17-20)}$.

El término estrés se volvió popular debido a que a Selye le gustaba interactuar con los medios. Le atraían los reflectores, le encantaba "mojar prensa", pocos como él podían manipularla; era una luminaria. Uno de sus discípulos, el argentino Pasqualini, dice lo siguiente: "Las conferencias de Selye eran teatrales. Pude presenciar en varias oportunidades su demostración del efecto anestésico de las hormonas. Aparecía con un ratón blanco en el bolsillo y un jarrón de peces rojos en una mano. En cuanto comenzaba a hablar, inyectaba al ratón con la hormona y lo dejaba sobre la mesa, al tiempo que esparcía polvo de otra hormona sobre el agua de los peces. Al momento señalado, el ratón caía dormido y los peces comenzaban a flotar sobre la superficie del agua de la pecera. Selye tenía, sin duda, una personalidad carismática y absorbente, lo cual le hacía ganar grandes amigos y no pocos enemigos"(21,22).

Por supuesto, él no fue el único con estas características; también Christian Barnard - padre de los trasplantes cardiacos- que parecía un actor por el histrionismo que le imprimía a sus conferencias, pues al mostrar sus geniales manos, ya deformadas por la artritis reumatoide, finalizaba con su voz "en crescendo" y con lágrimas en sus ojos, algo que hacía inevitable la ovación del público, hipnotizado por sus relatos. En nuestro medio, este estilo no ha sido ajeno a Manuel Elkin Patarroyo, e incluso a Rodolfo Llinás en sus últimos años. Pero - volviendo al estrés - no hay duda de que el concepto es fascinante, es fácil entenderlo como explicación de las dificultades y sufrimientos cotidianos, es decir, se trata de una acepción "taquillera". Libros de Selye como Stress without distress y The stress of life fueron éxitos editoriales en su época ${ }^{(8,10)}$.

Estos dos libros mezclan datos de sus investigaciones con postulaciones filosóficas que indican la forma de obtener la felicidad en la vida. Nada nuevo en cuanto a que ya se ha hablado del Yin y el Yang, la división maniquea de buenos y malos, la visión cristiana del perdón y del amor versus la ley del Talión, el antagonismo de placer y el sufrimiento, la abundancia y la escasez, el poder y la esclavitud, el macho que domina a la hembra y a sus súbditos. La felicidad (o el bienestar) de los países se puede medir con indicadores como el "Gini" que difieren del producto interno bruto y su distribución (a menudo inequitativa). Con su endocrinología social, Selye postula que la felicidad se obtiene gracias a un "egoísmo altruista" que promueve la interdependencia de los seres humanos y que evita un gasto rápido y excesivo de la "energía adaptativa" ante la incapacidad de manejar satisfactoriamente el estrés cotidiano que conduce a un envejecimiento prematuro y a la enfermedad. La fisiología endocrina al servicio de la salud mental, la felicidad obtenida en virtud de un acercamiento a lo que ocurre en la naturaleza.

Es usual que el adulto con estrés y depresión tenga antecedentes traumáticos en la niñez. Entre ellos podemos anotar la violencia intrafamiliar, el abuso físico y sexual, las carencias afectivas y nutricionales, la separación de los padres, la falta de educación y de oportunidades, el embarazo adolescente, el inicio sexual y toxicomanías tempranas, lo que lleva a un círculo vicioso en la descendencia del adulto con estrés ${ }^{(19,20)}$.

Al parecer, la red de genes involucrados en la adaptación y la supervivencia son adecuados para el hombre primitivo -donde la vida estaba diariamente en riesgo- pero inadecuados para el hombre moderno. Para responder a la amenaza a la supervivencia hay que combatir la desnutrición, la deshidratación, las infecciones, minimizar la exposición al peligro, anticiparse al enemigo y prevenir las lesiones tisulares. Se trata de conservar la energía, las proteínas, las calorías, los líquidos y electrolitos, potenciar el sistema inmune. Pero en la actualidad, el estrés crónico o biopsicosocial lleva a obesidad y síndrome metabólico con sedentarismo, a hipertensión y baja en las defensas, a las alergias, a la ansiedad, a depresión e insomnio, a las toxicomanías, a la fatiga crónica, a enfermedades típicas del desarrollo. El hombre primitivo, para conseguir una comida frugal y con mucha fibra tenía que realizar mucho ejercicio físico, que le impedía el sobrepeso y lo mantenía activo. Pero moría luchando, en las guerras tribales o en las sequías y fenómenos naturales. Según Selye, es necesario dosificar el gasto de la energía adaptativa, evitando las luchas inútiles, quizá comprando la tranquilidad. Además, no es solo el hombre el que está enfermo; de manera análoga también lo está la sociedad $^{(18)}$.

Dice Jackson: "A pesar de que pensadores como Huxley, Stephens o Ingle expresen sus reservas acerca de la aplicabilidad de los principios biológicos a los problemas sociopolíticos, varias características de la analogía orgánica siguen siendo conceptos fértiles no solo para los científicos sino para analistas sociales y para novelistas". El análisis de estos fenómenos encierra algo de verdad pero también algo de ficción ${ }^{(23)}$. 
Figura 1. Hans Selye, Efraim Otero Ruiz y Hernán Mendoza Hoyos, en el Club Médico de Bogotá.

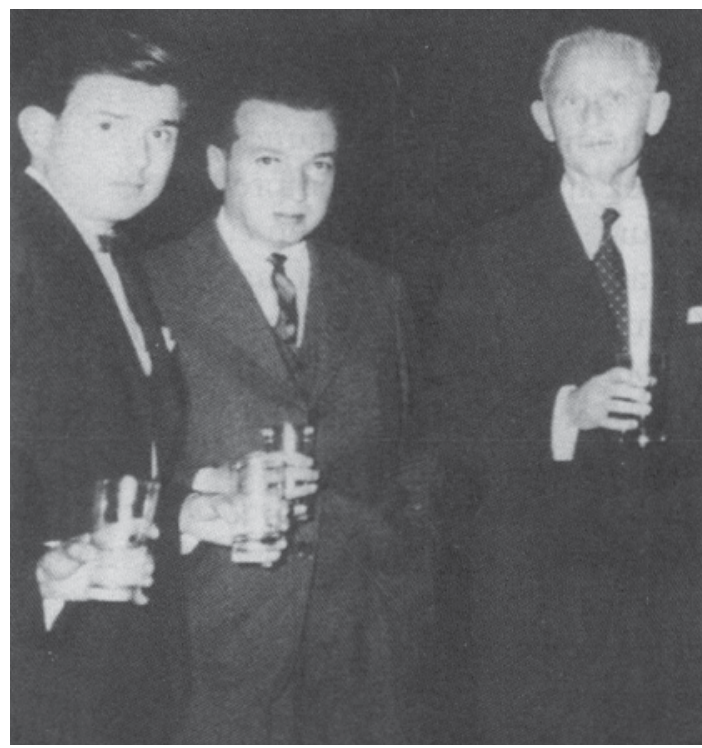

\section{Selye y la calcifilaxia}

En 1961, Selye construyó un modelo experimental en el que pudo precipitar una calcificación sistémica en ratas nefrectomizadas, algo parecido a lo que ocurre con un síndrome que habían informado Bryant y White en $1898^{(25)}$. La calcifilaxia - término acuñado por Selye- es una enfermedad de causa desconocida asociada al depósito de calcio en las paredes de los vasos de pequeño y mediano calibre, con la consiguiente necrosis isquémica progresiva y gangrena del territorio que irrigan, con alta mortalidad. Esta calcinosis rara tiene importancia clínica en pacientes urémicos y en hemodiálisis, definiéndola como una hipersensibilidad inducida por un grupo de agentes sensibilizantes, cuando además está presente un grupo de estimuladores después de un periodo crítico de tiempo. Por aquella época precisamente, Selye visitó a Colombia (figura 1). Sus conferencias quedaron plasmadas en un número de la revista de la Sociedad (con carátula blanca y fotografías a color de los ratones de experimentación), que incluyó diversas investigaciones suyas sobre calcifilaxia en ratones, calcificación órgano-específica relacionada con nefrectomía, hormona paratiroidea o vitamina D en exceso. También son agentes estimuladores de la calcificación sistémica la albúmina de huevo y las sales de metales. Después se han informado muchos casos que han ampliado la documentación sobre este fenómeno relacionado con el hiperparatiroidismo secundario e hipercalcemia que se ve en los urémicos y que mejora con calcitriol. Esta calcifilaxia parece ser diferente a las metástasis cálcicas observadas en el síndrome.

Aunque el austro-canadiense investigó muchos tópicos, sin duda el lanzamiento de su concepto sobre estrés biológico fue su mayor aporte a la humanidad.

\section{Referencias}

1. Selye H. A syndrome produced by diverse nocuous agents. Nature 1936; 138:32.

2. Selye H. Thymus and adrenals in the response of the organism to injuries and intoxication. Brit J Exp Path 1936; 17: 234-48

3. Cannon WB., Pereira JR. Increase of Adrenal Secretion in Fever. Proc Natl Acad Sci USA. 1924; 10:247-248.

4. Cannon WB. Stresses and strains of homeostasis. Am. J. Med. Sci. 1935; 189:1-4.

5. Jácome-Roca A. Historia de lass Hormonas. Academia Nacional de Medicina, Bogotá. 2005.

6. http://es.wikipedia.org/wiki/Hans_Selye

7. Goldstein DS, Kopin IJ. Evolution of concepts of stress. Stress. 2007;10(2):109-20.

8. Selye H. The stress of my life. A scientist's memoirs. Toronto: McClelland \& Stewart, 1977

9. The legacy of Hans Selye and the origins of stress research: A retrospective 75 years after his landmark brief "Letter" to the Editor of Nature Stress. 2012 15 (5): 472-478.

10. Selye H. Stress sans détresse. Montréal: Les Éditions La Presse, 1974.

11. Selye H. Morphological changes in the fowl following chronic overdosage with various steroids. J Morph 1943; 73: 401-21.

12. Selye $H$. The general adaptation syndrome and the diseases of adaptation. J Clin Endocrinol 1946; 6: 117-230.
13. Selye, H. Pharmacological classification of steroid hormones. Nature 1941 148,84-85.

14. Selye H. (1941). Anaesthetic effects of steroid hormones. Proc. Soc. Exp. Biol $46,116-121$.

15. Nobel Prize.

16. McEwen BS. Stressed or stressed out: what is the difference? J Psychiatry Neurosci 2005; 30: 315-318.

17. Le Moal M. Historical approach and evolution of the stress concept: a persona account. Psychoneuroendocrinol. 2007;32 Suppl 1:S3-9.

18. Jácome-Roca A. Estrés y enfermedad, Enfoque psiconeuroinmunoendocrino Medicina (Bogotá) 2010; 32 (3): 233-242.

19. Trucco M. Estrés y trastornos mentales, aspectos neurobiológicos y psicosociales. Rev Chil Neuropsiquiatr 2002; 40 (S2): 8-19.

20. Swaab DF,Bao AM, Lucassen PJ. The stress system in the human brain in depression and neurodegeneration. Ageing Res Rev 2005; 4: 141-194.

21. Pasqualini CD. Stress y resiliencia, Hans Selye y el encuentro de las dos culturas Medicina (Buenos Aires) 2013; 73: 504-505.

22. Pasqualini CD. Lainvestigación básica según Hans Selye MEDICINA (Buenos Aires) 2007; 67: 767-770.

23. Jackson M. The pursuit of happiness, The social and scientific origins of Hans Selye's natural philosophy of life. Hist Human Sci. Dec 2012; 25(5): 13-21.

24. Weenig RH. Pathogenesis of calciphylaxis: Hans Selye to nuclear factor kappaB. J Am Acad Dermatol. 2008;58(3):458-71.

25. Selye, H., Gentile, G., Prioreschi, P. (1961) Calciphylaxis: Cutaneous molt in duced by calciphylaxis in the rat. Science 134,1876-1877. 